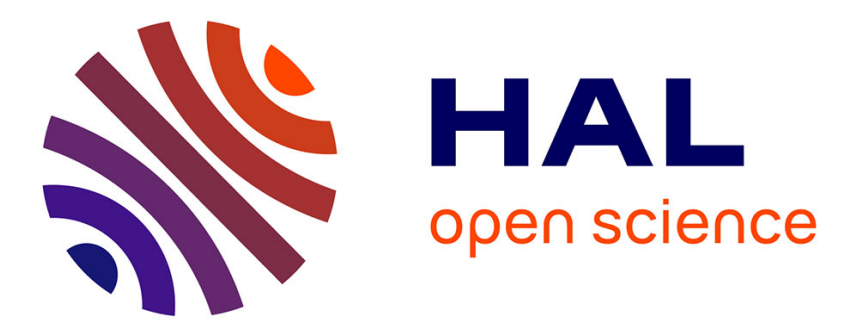

\title{
Influence of composition and thermal history of volcanic glasses on water content as determined by micro-Raman spectrometry
}

Andrea Di Muro, D. Giordano, B. Villemant, Gilles Montagnac, Bruno

Scaillet, C. Romano

\section{To cite this version:}

Andrea Di Muro, D. Giordano, B. Villemant, Gilles Montagnac, Bruno Scaillet, et al.. Influence of composition and thermal history of volcanic glasses on water content as determined by micro-Raman spectrometry. Applied Geochemistry, 2006, 21 (5), pp.802-812. hal-00022621

\section{HAL Id: hal-00022621 \\ https://hal.science/hal-00022621}

Submitted on 24 May 2006

HAL is a multi-disciplinary open access archive for the deposit and dissemination of scientific research documents, whether they are published or not. The documents may come from teaching and research institutions in France or abroad, or from public or private research centers.
L'archive ouverte pluridisciplinaire HAL, est destinée au dépôt et à la diffusion de documents scientifiques de niveau recherche, publiés ou non, émanant des établissements d'enseignement et de recherche français ou étrangers, des laboratoires publics ou privés. 


\title{
Influence of composition and thermal history of volcanic glasses on water content as determined by micro-Raman spectrometry ${ }^{\text {th }}$
}

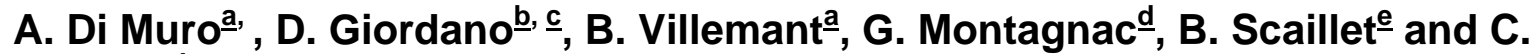 \\ Romano $\underline{\text { b }}$
}

a Laboratoire de Physique et Chimie des Systèmes Volcaniques, IPGP-UPMC, CNRS-UMR 7046, Place Jussieu 4, 75005, Paris, France

${ }^{b}$ Dipartimento di Scienze Geologiche, Università Roma Tre, L.go S.L. Murialdo, 1, 00154 Rome, Italy

${ }^{c}$ Department of Earth and Ocean Sciences, University of British Columbia, 6339

Stores Road, V6T 1X7, Vancouver B.C., Canada

${ }^{d}$ Laboratoire de Sciences de la Terre, Ecole Normale Supérieure, 46 Allée d'Italie, 69364 ,Lyon, France

${ }^{e}$ Institut des Sciences de la Terre d'Orléans-CNRS-UO, 1A, rue de la Férollerie 45071, Orléans, France

\section{Abstract}

Development of Raman spectrometry for quantification of water content in natural glasses requires the assessment of the dependence of the technique on glass composition and thermal history. In the low frequency domain, Raman spectra topology varies due to glass depolymerization and substitution in the framework of $\left(\mathrm{Si}^{4+}\right)^{\mathrm{IV}}$ by alkali-balanced $\left(\mathrm{Al}^{3+}\right)^{\mathrm{IV}}$ and $\left(\mathrm{Fe}^{3+}\right)^{\mathrm{IV}}$ in calcalkaline (rhyolite to basaltic andesite) and alkaline (trachyte, phonolite to alkali basalt) glasses. These processes result in strong dependence of previous analytical procedure (internal calibration) on glass composition. Here, we show that an analytical procedure based on calibration to an external standard is only faintly composition-dependent for Si-rich alkaline glasses (trachytes-phonolites). For a given glass composition, thermal history also plays a fundamental role in the choice of Raman procedure for water analysis. Repeated cycles of thermal annealing induce microcrystallization of hydrous trachyte glasses and modify cation distribution in the glass structure. Application of these concepts to analysis of banded obsidians suggests that small-scale heterogeneities in glasses are not simply related to magma degassing, but could depend on thermal history and consequent relaxation paths in the melt.

Keywords: Raman; Glasses; Alkaline magmas; Volatiles; Water; Obsidian; Viscous dissipation 


\section{Introduction}

Hydrous volcanic glasses often exhibit chemical and physical (e.g. crystallinity, vesicularity, color) heterogeneities even at micrometric scale. In these materials, accurate and fast analysis of total dissolved water content $\left(\mathrm{H}_{2} \mathrm{O}_{\mathrm{T}}\right)$ and of water speciation $\left(\mathrm{H}_{2} \mathrm{O}_{m} / \mathrm{OH}\right)$ requires the development of micro-analytical techniques able to combine high spatial resolution with no or low compositional dependence. MicroRaman spectrometry allows probing glass volumes $<10 \mu \mathrm{m}^{3}$ and recent development of this technique shows that, for well-chosen analytical procedures, it has a moderate dependence on major element contents (Di Muro et al., 2006).

For calibration of Raman analytical procedure, height or area of the $\mathrm{H}_{2} \mathrm{O}_{\mathrm{T}}$ band $(\sim$ $3.550 \mathrm{~cm}^{-1}$ ) is normalized to other reference bands and correlated with the amount of water dissolved in a given sample. Water band can be normalized either to TOT bands ( $T$ : tetrahedrally coordinated cations; $\mathrm{O}$ : bridging oxygen) related to vibrations of the aluminosilicate framework (internal normalization procedure; Thomas, 2000 and Chabiron et al., 2004) or to total $\mathrm{OH}$-stretching band of a glass standard (external normalization procedure; Thomas, 2000 and Di Muro et al., 2006). As a consequence, it is expected that Raman calibration routines are potentially dependent on physico-chemical processes affecting glass structure and water speciation (Dingwell and Virgo, 1987, Zhang et al., 1995, Romano et al., 2003 and Giordano et al., 2004). Changes in glass structure affect the spectral composition of the TOT bands (Furukawa et al., 1981, Mysen, 1988 and Sharma et al., 1997). In turn, water speciation influences the geometry and the spectral distribution of $\mathrm{H}_{2} \mathrm{O}_{\mathrm{T}}$ band (Chabiron et al., 2004 and Di Muro et al., 2006). Furthermore, previous researches have preferentially characterized hydrous synthetic glasses of simple composition (Mysen et al., 1980; Mysen and Virgo, 1986) or silica-rich glasses (quartz, rhyolites) (McMillan and Remmele, 1986, Thomas, 2000 and Chabiron et al., 2004). Finally, melt inclusions in crystals and groundmass melts can experience complex thermal paths in magma reservoir (Bachmann and Bergantz, 2003), during ascent in the conduit (Rosi et al., 2004) and during emplacement (Gottsmann and Dingwell, 2002) or simply during sample preparation (e.g. heating for homogenization of glass inclusions or heating stages during the viscosity measurements).

In this frame, the influence of glass composition and thermal history represent critical issues for development of Raman spectrometry for water measurement. This paper is a contribution to assess the influence of complex glass chemistry and cooling conditions on Raman routines for water analysis in natural samples. We have tested Raman procedures for $\mathrm{H}_{2} \mathrm{O}_{\mathrm{T}}$ determination on natural alkali-rich aluminosilicate glasses from Phlegrean Fields (MNV trachyte) and Mt. Vesuvius (Pm and Po phonolites). MNV composition is that of the melt of the last historical eruption (AD 1538) of Phlegrean Fields (Monte Nuovo; details are in Giordano et al., 2004). Two sets of MNV glasses with different thermal histories and variable $\mathrm{H}_{2} \mathrm{O}$ content have been studied to determine the influence of thermal annealing on Raman analyses. $\mathrm{Pm}$ (Ca,Fe-poor) and $\mathrm{Po}$ (Ca,Fe-rich) compositions are respectively those of the melt fraction of the magmas erupted during $79 \mathrm{AD}$ Pompei and $472 \mathrm{AD}$ Pollena plinian eruptions of Mt. Vesuvius and were characterized in a previous research (Di Muro et al., 2006). 


\section{Methodology}

\subsection{Glass samples synthesis}

In order to obtain crystal- and bubble-free dry glasses ( $<200$ ppm $\left.\mathrm{H}_{2} \mathrm{O}_{\mathrm{T}}\right)$, starting natural samples (pumice matrixes) were powdered, melted in Pt crucibles in a $\mathrm{MoSi}_{2}$ box furnace at $T: 1450-1600{ }^{\circ} \mathrm{C}$ (trachytes) or $1200{ }^{\circ} \mathrm{C}$ (phonolites) and finally quenched in air or by pouring them on a steel plate. Visual and BSE inspection indicated that obtained glasses were crystal- and bubble-free.

To synthesize hydrous glass sets, powders of the anhydrous glasses were loaded together with known amounts of doubly distilled water in Pt capsules (outer diameter $5.2 \mathrm{~mm}$, wall thickness $0.1 \mathrm{~mm}$ and length $14-15 \mathrm{~mm}$ ) and then sealed by arcwelding. In order to control possible water leakage, capsules were dried in an oven at $110{ }^{\circ} \mathrm{C}$ for at least 1 hour and checked for weight loss. This ensured a homogeneous distribution of water inside the capsules. Trachyte glasses were hydrated in a piston cylinder apparatus at $P$ : $10 \mathrm{kbar}$ pressure and $T: 1450-1600{ }^{\circ} \mathrm{C}$ and then rapidly quenched (initial quench rates: ca. $200 \mathrm{~K} / \mathrm{s}$ ). Phonolite glasses were hydrated at lower pressure $(2 \mathrm{kbar})$ and temperature $\left(1200^{\circ} \mathrm{C}\right)$. Total dissolved water in hydrous glasses was determined by Karl Fisher Titration (KFT).

A subset of the trachyte glasses, having variable water contents $(0.02-3.86 \mathrm{wt} \%)$, experienced a further heating-cooling cycle. Here, we report the most salient features relative to the thermal stages of the adopted procedure. Trachyte samples were heated under an $\mathrm{Ar}$ atmosphere at a constant heating rate of $10 \mathrm{~K} / \mathrm{min}$ until a temperature 140-200 K lower than the glass transition (Giordano et al., 2004). Subsequent heating had a rate of $5 \mathrm{~K} / \mathrm{min}$ up to attaining the target temperature (Table 1) at which the samples were allowed to structurally relax during an isothermal dwell (Dingwell et al., 1996). Isothermal dwells of 15-20 min were typically adopted for thin hydrous samples ( $\sim 1 \mathrm{~mm}$ thick), whereas a longer time of about $1 \mathrm{~h}$ was necessary for the relaxation of the larger dry samples $(\sim 2-3 \mathrm{~mm}$ thick, up to $6 \mathrm{~mm}$ in diameter) (Giordano et al., 2004).

EPMA (major elements and $\mathrm{Cl}$ ) and FTIR (water) analyses were performed before and after heating to check any chemical drift. Results of the EPMA analyses are presented in Table 1 together with the water content determined by KFT. Close inspection of data sets reveals that fusion and thermal annealing of glasses with up to $3.9 \mathrm{wt} \% \mathrm{H}_{2} \mathrm{O}_{\mathrm{T}}$ have not modified glass chemistry. In particular, neither water- nor Cl-losses have been observed in these alkali-rich melts (Table 1). 
Table 1. : Composition (EPMA) and synthesis conditions of the hydrous glass sets studied in this paper

\begin{tabular}{|c|c|c|c|c|c|c|c|c|c|c|c|c|c|c|c|c|}
\hline $\begin{array}{l}\text { Samp } \\
\text { le }\end{array}$ & $\begin{array}{l}79 A \\
D\end{array}$ & & $\begin{array}{l}472 \\
A D\end{array}$ & & $\begin{array}{l}\text { MNV } \\
-0\end{array}$ & & $\begin{array}{l}\text { MNV } \\
-0^{*}\end{array}$ & & $\begin{array}{l}\text { MNV } \\
806\end{array}$ & & $\begin{array}{l}\text { MNV } \\
806^{*}\end{array}$ & & $\begin{array}{l}\text { MNV } \\
804^{*}\end{array}$ & & $\begin{array}{l}\text { MNV } \\
2166\end{array}$ & \\
\hline $\begin{array}{l}\text { Prove } \\
\text { nance }\end{array}$ & $\begin{array}{l}\text { Ves } \\
\text { uviu } \\
\text { s }\end{array}$ & & $\begin{array}{l}\text { Ves } \\
\text { uviu } \\
\text { s }\end{array}$ & & $\begin{array}{l}\text { Phle } \\
\text { grea } \\
\mathrm{n} \\
\text { Field } \\
\mathrm{s}\end{array}$ & & $\begin{array}{l}\text { Phle } \\
\text { grea } \\
\text { n } \\
\text { Field } \\
\text { s }\end{array}$ & & $\begin{array}{l}\text { Phle } \\
\text { grea } \\
\text { n } \\
\text { Field } \\
\text { s }\end{array}$ & & $\begin{array}{l}\text { Phle } \\
\text { grea } \\
\text { n } \\
\text { Field } \\
\text { s }\end{array}$ & & $\begin{array}{l}\text { Phle } \\
\text { grea } \\
\mathrm{n} \\
\text { Field } \\
\mathrm{s}\end{array}$ & & $\begin{array}{l}\text { Phle } \\
\text { grea } \\
\mathrm{n} \\
\text { Field } \\
\mathrm{s}\end{array}$ & \\
\hline $\begin{array}{l}\text { Refer } \\
\text { ence }\end{array}$ & 1 & & 1 & & 2 & & 2 & & 2 & & 2 & & 2 & & 2 & \\
\hline $\begin{array}{l}\text { Comp } \\
\text { ositio } \\
\text { n }\end{array}$ & $\begin{array}{l}\text { L- } \\
\text { Ca } \\
\text { Pota } \\
\text { ssic } \\
\text { Pho } \\
\text { nolit } \\
\text { e }\end{array}$ & & $\begin{array}{l}\mathrm{H}- \\
\mathrm{Ca} \\
\text { Pota } \\
\text { ssic } \\
\text { Pho } \\
\text { nolit } \\
\text { e }\end{array}$ & & $\begin{array}{l}\text { Trac } \\
\text { hyte }\end{array}$ & & $\begin{array}{l}\text { Trac } \\
\text { hyte }\end{array}$ & & $\begin{array}{l}\text { Trac } \\
\text { hyte }\end{array}$ & & $\begin{array}{l}\text { Trac } \\
\text { hyte }\end{array}$ & & $\begin{array}{l}\text { Trac } \\
\text { hyte }\end{array}$ & & $\begin{array}{l}\text { Trac } \\
\text { hyte }\end{array}$ & \\
\hline $\begin{array}{l}\mathrm{H}_{2} \mathrm{O} \\
\text { (rang } \\
\mathrm{e} \\
\mathrm{wt} \% \text { ) }\end{array}$ & $\begin{array}{l}0.02 \\
\overline{6} .80\end{array}$ & & $\begin{array}{l}0.02 \\
\overline{6} .70\end{array}$ & & 0.02 & & 0.02 & & 1.39 & & 1.39 & & 1.00 & & 3.86 & \\
\hline $\begin{array}{l}n \\
\text { Analy } \\
\text { ses }\end{array}$ & 8 & $\begin{array}{l}1 \\
\sigma\end{array}$ & 8 & $\begin{array}{l}1 \\
\sigma\end{array}$ & 4 & $\begin{array}{l}1 \\
\sigma\end{array}$ & 2 & $\begin{array}{l}1 \\
\sigma\end{array}$ & 3 & $\begin{array}{l}1 \\
\sigma\end{array}$ & 3 & $\begin{array}{l}1 \\
\sigma\end{array}$ & 3 & $\begin{array}{l}1 \\
\sigma\end{array}$ & 3 & $\begin{array}{l}1 \\
\sigma\end{array}$ \\
\hline $\mathrm{SiO}_{2}$ & $\begin{array}{l}56.0 \\
9\end{array}$ & $\begin{array}{l}0 . \\
3 \\
7\end{array}$ & $\begin{array}{l}51.3 \\
6\end{array}$ & $\begin{array}{l}0 . \\
3 \\
5\end{array}$ & $\begin{array}{l}63.2 \\
9\end{array}$ & $\begin{array}{l}0 . \\
3 \\
4\end{array}$ & $\begin{array}{l}63.2 \\
7\end{array}$ & $\begin{array}{l}0 . \\
1 \\
0\end{array}$ & $\begin{array}{l}63.5 \\
5\end{array}$ & $\begin{array}{l}0 . \\
4 \\
4\end{array}$ & $\begin{array}{l}63.7 \\
8\end{array}$ & $\begin{array}{l}0 . \\
3 \\
6\end{array}$ & $\begin{array}{l}63.8 \\
7\end{array}$ & $\begin{array}{l}0 . \\
2 \\
5\end{array}$ & $\begin{array}{l}63.4 \\
0\end{array}$ & $\begin{array}{l}0 . \\
1 \\
6\end{array}$ \\
\hline $\mathrm{TiO}_{2}$ & 0.19 & $\begin{array}{l}0 . \\
0 \\
5\end{array}$ & 0.48 & $\begin{array}{l}0 . \\
0 \\
7\end{array}$ & 0.45 & $\begin{array}{l}0 . \\
0 \\
2\end{array}$ & 0.41 & $\begin{array}{l}0 . \\
0 \\
5\end{array}$ & 0.43 & $\begin{array}{l}0 . \\
0 \\
3\end{array}$ & 0.43 & $\begin{array}{l}0 . \\
0 \\
1\end{array}$ & 0.46 & $\begin{array}{l}0 . \\
0 \\
0\end{array}$ & 0.45 & $\begin{array}{l}0 . \\
0 \\
6\end{array}$ \\
\hline $\mathrm{Al}_{2} \mathrm{O}_{3}$ & $\begin{array}{l}22.0 \\
2\end{array}$ & $\begin{array}{l}0 . \\
1 \\
5\end{array}$ & $\begin{array}{l}21.6 \\
3\end{array}$ & $\begin{array}{l}0 . \\
2\end{array}$ & $\begin{array}{l}18.3 \\
0\end{array}$ & $\begin{array}{l}0 . \\
1 \\
7\end{array}$ & $\begin{array}{l}18.1 \\
2\end{array}$ & $\begin{array}{l}0 . \\
0 \\
9\end{array}$ & $\begin{array}{l}18.2 \\
2\end{array}$ & $\begin{array}{l}0 . \\
1 \\
0\end{array}$ & $\begin{array}{l}18.1 \\
5\end{array}$ & $\begin{array}{l}0 . \\
2 \\
1\end{array}$ & $\begin{array}{l}18.0 \\
7\end{array}$ & $\begin{array}{l}0 . \\
0 \\
9\end{array}$ & $\begin{array}{l}18.1 \\
2\end{array}$ & $\begin{array}{l}0 . \\
0 \\
6\end{array}$ \\
\hline $\mathrm{FeOa}$ & 2.26 & $\begin{array}{l}0 . \\
3 \\
1\end{array}$ & 4.54 & $\begin{array}{l}0 . \\
2 \\
3\end{array}$ & 3.24 & $\begin{array}{l}0 . \\
0 \\
9\end{array}$ & 3.14 & $\begin{array}{l}0 . \\
0 \\
7\end{array}$ & 3.20 & $\begin{array}{l}0 . \\
0 \\
8\end{array}$ & 3.33 & $\begin{array}{l}0 . \\
0 \\
1\end{array}$ & 3.21 & $\begin{array}{l}0 . \\
1 \\
0\end{array}$ & 3.21 & $\begin{array}{l}0 . \\
0 \\
3\end{array}$ \\
\hline $\mathrm{MnO}$ & nd & & nd & & 0.21 & $\begin{array}{l}0 . \\
0 \\
2\end{array}$ & 0.22 & $\begin{array}{l}0 . \\
0 \\
0\end{array}$ & 0.21 & $\begin{array}{l}0 . \\
0 \\
2\end{array}$ & 0.20 & $\begin{array}{l}0 . \\
0 \\
1\end{array}$ & 0.20 & $\begin{array}{l}0 . \\
0 \\
2\end{array}$ & 0.20 & $\begin{array}{l}0 . \\
0 \\
1\end{array}$ \\
\hline $\mathrm{MgO}$ & 0.18 & $\begin{array}{l}0 . \\
0 \\
4\end{array}$ & 0.74 & $\begin{array}{l}0 . \\
0 \\
5\end{array}$ & 0.37 & $\begin{array}{l}0 . \\
0 \\
3\end{array}$ & 0.37 & $\begin{array}{l}0 . \\
0 \\
0\end{array}$ & 0.41 & $\begin{array}{l}0 . \\
0 \\
2\end{array}$ & 0.41 & $\begin{array}{l}0 . \\
0 \\
3\end{array}$ & 0.43 & $\begin{array}{l}0 . \\
0 \\
0\end{array}$ & 0.42 & $\begin{array}{l}0 . \\
0 \\
2\end{array}$ \\
\hline $\mathrm{CaO}$ & 2.80 & $\begin{array}{l}0 . \\
1 \\
3\end{array}$ & 5.90 & $\begin{array}{l}0 . \\
1 \\
1\end{array}$ & 1.90 & $\begin{array}{l}0 . \\
0 \\
5\end{array}$ & 1.93 & $\begin{array}{l}0 . \\
0 \\
3\end{array}$ & 1.85 & $\begin{array}{l}0 . \\
0 \\
3\end{array}$ & 1.81 & $\begin{array}{l}0 . \\
0 \\
5\end{array}$ & 1.83 & $\begin{array}{l}0 . \\
0 \\
9\end{array}$ & 1.79 & $\begin{array}{l}0 . \\
0 \\
3\end{array}$ \\
\hline
\end{tabular}




\begin{tabular}{|c|c|c|c|c|c|c|c|c|c|c|c|c|c|c|c|c|}
\hline $\mathrm{Na}_{2} \mathrm{O}$ & 6.22 & $\begin{array}{l}0 . \\
2 \\
1\end{array}$ & 5.92 & $\begin{array}{l}0 . \\
0 \\
8\end{array}$ & 5.07 & $\begin{array}{l}0 . \\
0 \\
5\end{array}$ & 5.26 & $\begin{array}{l}0 . \\
0 \\
2\end{array}$ & 4.98 & $\begin{array}{l}0 . \\
1 \\
7\end{array}$ & 4.91 & $\begin{array}{l}0 . \\
1 \\
3\end{array}$ & 4.91 & $\begin{array}{l}0 . \\
1 \\
4\end{array}$ & $\begin{array}{l}5.24 \\
+\end{array}$ & $\begin{array}{l}0 . \\
1 \\
9\end{array}$ \\
\hline $\mathrm{K}_{2} \mathrm{O}$ & $\begin{array}{l}10.2 \\
5\end{array}$ & $\begin{array}{l}0 . \\
1 \\
8\end{array}$ & 9.42 & $\begin{array}{l}0 . \\
1 \\
6\end{array}$ & 7.17 & $\begin{array}{l}0 . \\
1 \\
5\end{array}$ & 7.29 & $\begin{array}{l}0 . \\
0 \\
0\end{array}$ & 7.17 & $\begin{array}{l}0 . \\
1 \\
9\end{array}$ & 6.97 & $\begin{array}{l}0 . \\
0 \\
9\end{array}$ & 7.01 & $\begin{array}{l}0 . \\
0 \\
4\end{array}$ & $\begin{array}{l}7.18 \\
+\end{array}$ & $\begin{array}{l}0 . \\
1 \\
8\end{array}$ \\
\hline $\begin{array}{l}\text { Clpp } \\
\text { m }\end{array}$ & nd & & nd & & 3300 & $\begin{array}{l}1 \\
1 \\
0\end{array}$ & 3430 & $\begin{array}{l}9 \\
0\end{array}$ & 3500 & $\begin{array}{l}1 \\
4 \\
0\end{array}$ & 3490 & $\begin{array}{l}6 \\
0\end{array}$ & 3540 & $\begin{array}{l}3 \\
0\end{array}$ & 3430 & $\begin{array}{l}1 \\
8 \\
0\end{array}$ \\
\hline Total & 99 & $\begin{array}{l}0 . \\
5 \\
0\end{array}$ & 98 & $\begin{array}{l}0 . \\
6 \\
0\end{array}$ & $\begin{array}{l}99.0 \\
2\end{array}$ & $\begin{array}{l}0 . \\
9 \\
7\end{array}$ & $\begin{array}{l}98.7 \\
9\end{array}$ & $\begin{array}{l}0 . \\
0 \\
7\end{array}$ & $\begin{array}{l}98.0 \\
6\end{array}$ & $\begin{array}{l}0 . \\
2 \\
1\end{array}$ & $\begin{array}{l}98.4 \\
4\end{array}$ & $\begin{array}{l}0 . \\
6 \\
0\end{array}$ & $\begin{array}{l}98.4 \\
3\end{array}$ & $\begin{array}{l}0 . \\
5 \\
0\end{array}$ & $\begin{array}{l}96.6 \\
4\end{array}$ & $\begin{array}{l}0 . \\
3 \\
7\end{array}$ \\
\hline $\begin{array}{l}\mathrm{NBO} / \\
\mathrm{T} \\
\text { (rang } \\
\text { e) }\end{array}$ & $\begin{array}{l}0.08 \\
\overline{0} \\
0.63\end{array}$ & & $\begin{array}{l}0.19 \\
- \\
0.76\end{array}$ & & 0.05 & & 0.06 & & 0.16 & & 0.15 & & 0.13 & & 0.35 & \\
\hline $\begin{array}{l}\mathrm{A} / \mathrm{CN} \\
\mathrm{K}^{\mathrm{a}}\end{array}$ & 0.83 & & 0.71 & & 0.94 & & 0.90 & & 0.94 & & 0.96 & & 0.95 & & 0.92 & \\
\hline $\begin{array}{l}\mathrm{Na}_{2} \mathrm{O} \\
+\mathrm{K}_{2} \mathrm{O}\end{array}$ & 16.4 & & 15.3 & & 12.2 & & 12.5 & & 12.1 & & 11.9 & & 11.9 & & 12.4 & \\
\hline $\begin{array}{l}\mathrm{K}_{2} \mathrm{O} / \\
\mathrm{Na}_{2} \mathrm{O}\end{array}$ & 1.6 & & 1.6 & & 1.4 & & 1.4 & & 1.4 & & 1.4 & & 1.4 & & 1.4 & \\
\hline $\begin{array}{l}\mathrm{Al} /(\mathrm{Al} \\
+\mathrm{Si})\end{array}$ & 0.32 & & 0.33 & & 0.25 & & 0.25 & & 0.25 & & 0.25 & & 0.25 & & 0.25 & \\
\hline \multicolumn{17}{|c|}{ Glass synthesis conditions } \\
\hline $\begin{array}{l}\text { Techn } \\
\text { ique }\end{array}$ & IHV & & IHV & & PC & & PC & & PC & & PC & & PC & & PC & \\
\hline $\begin{array}{l}P \\
\text { (kbar) }\end{array}$ & 2 & & 2 & & 10 & & 10 & & 10 & & 10 & & 10 & & 10 & \\
\hline$T\left({ }^{\circ} \mathrm{C}\right)$ & $\begin{array}{l}130 \\
0\end{array}$ & & $\begin{array}{l}130 \\
0\end{array}$ & & $\begin{array}{l}1450 \\
- \\
1600\end{array}$ & & $\begin{array}{l}1450 \\
- \\
1600\end{array}$ & & $\begin{array}{l}1450 \\
- \\
1600\end{array}$ & & $\begin{array}{l}1450 \\
- \\
1600\end{array}$ & & $\begin{array}{l}1450 \\
- \\
1600\end{array}$ & & $\begin{array}{l}1450 \\
- \\
1600\end{array}$ & \\
\hline $\begin{array}{l}\text { Quen } \\
\text { ch } \\
\text { rate } \\
\left({ }^{\circ} \mathrm{C} / \mathrm{s}\right)\end{array}$ & $\begin{array}{l}100 \\
- \\
200\end{array}$ & & $\begin{array}{l}100 \\
- \\
200\end{array}$ & & 200 & & 200 & & 200 & & 200 & & 200 & & 200 & \\
\hline $\begin{array}{l}T_{\text {anneali }} \\
\mathrm{ng}\left({ }^{\circ} \mathrm{C}\right)\end{array}$ & & & & & & & 740 & & & & 540 & & 580 & & 396 & \\
\hline
\end{tabular}

Reported water contents were determined by KFT. IHV: internally heated pressure vessel; PC: piston cylinder. NBO/T: non-bridging oxygen per tetrahedrally coordinated cation $\left(\mathrm{Si}, \mathrm{Al}, \mathrm{Fe}^{3+}, \mathrm{P}\right)$. Analytical conditions: $15.0 \mathrm{kv}, 10 \mathrm{nA}$, defocused beam of diameter $10-20 \mu \mathrm{m}$. Stars indicate glasses that have 
experienced thermal annealing after synthesis. References: 1: Di Muro et al., 2006; 2: Giordano et al., 2004.

${ }^{\mathrm{a}} \mathrm{Molar} \mathrm{Al}_{2} \mathrm{O}_{3} /\left(\mathrm{CaO}+\mathrm{Na}_{2} \mathrm{O}+\mathrm{K}_{2} \mathrm{O}\right)$.

\subsection{Raman spectrometry}

Raman scattering was excited using $514.5 \mathrm{~nm}$ wavelength of an argon ion laser from Spectra Physics and measurements were performed with a LabRam HR800 (ENSLyon) spectrometer manufactured Jobin-Yvon equipped with a Peltier-cooled CCD detector. Glasses were analyzed by focusing the laser beam on a $\sim 2 \mu \mathrm{m}$-wide spot under an Olympus microscope in confocal setting. The used pseudo-confocal system is characterized by low confocal performance (one pinhole) but high system throughput. To reduce sampling depth we adopted a small confocal hole $(100 \mu \mathrm{m})$ and focused through the objective with the highest magnification (100x). The same microscope objective permits observation of the sample, focuses the laser beam onto the surface of the sample and collects the scattered radiation (180 geometry). Spectra were obtained in the $150-2000 \mathrm{~cm}^{-1}$ (aluminosilicate framework domain, TOT; Fig. 2) and $3000-4000 \mathrm{~cm}^{-1}(\mathrm{OH}$-stretching domain) shift ranges relative to the exciting laser light. A grating of 600 grooves $/ \mathrm{mm}$ was used to cover in one scan each domain. That clearly resulted in moderate resolution $\left( \pm 4 \mathrm{~cm}^{-1}\right)$, but in an intense signal. Average laser power was about $200 \mathrm{~mW}$ and was reduced to $20 \mathrm{~mW}$ by $1 / 10$ filtering. Notch filter results in loss of about $20 \%$ on the beam power during its path. Laser power was slightly tuned to guarantee a constant laser power $(7.4 \mathrm{~mW})$ on the sample, which was periodically checked. In glasses containing $\mathrm{H}_{2} \mathrm{O}_{\top}>1 \mathrm{wt} \%$, acquisition times were of $3 \times 30 \mathrm{~s}$ for the high-frequencies domain and of $1 \times 30 \mathrm{~s}$ for the low frequencies. Longer counting times were adopted for glasses with $\mathrm{H}_{2} \mathrm{O}_{\mathrm{T}}<1 \mathrm{wt} \%$ to reduce background noise contribution. All spectra were obtained with the same optical configuration and 3-5 analyses were performed for each sample. Raman spectra are characterized in terms of band identification, band geometry (height, area, half-width) and band spectral composition after subtraction of a second order polynomial baseline. Detailed descriptions of spectra acquisition and treatment are reported in Di Muro et al. (2006).

\section{Calibration procedure for determination of dissolved water in glasses}

\subsection{Influence of glass composition}

In order to assess the influence of glass composition on the Raman normalization method, we collected room-temperature unpolarized spectra of volcanic samples having a composition ranging from Si-rich, Fe-poor rhyolites to increasingly Al- and Fe-rich, dacite, trachyte, phonolite, andesite, basaltic andesites and alkali basalt (Table 2). 
Table 2. : Composition of volcanic glasses analyzed in this study to assess the influence of complex composition on Raman spectra topology

\begin{tabular}{|c|c|c|c|c|c|c|c|c|c|c|c|c|}
\hline Sample & $\mathrm{TPh}$ & & Unz & & Pelée & & MST & & MRP & & ETN & \\
\hline $\begin{array}{l}\text { Provenan } \\
\text { ce }\end{array}$ & Teide & & $\begin{array}{l}\text { Unze } \\
\mathrm{n}\end{array}$ & & $\begin{array}{l}\text { Martini } \\
\text { ca }\end{array}$ & & $\begin{array}{l}\text { MontSer } \\
\text { rat }\end{array}$ & & Merapi & & Etna & \\
\hline $\begin{array}{l}\text { Referenc } \\
\mathrm{e}\end{array}$ & 3,4 & & 4 & & 4 & & 4 & & 4 & & 4,5 & \\
\hline $\begin{array}{l}\text { Compositi } \\
\text { on }\end{array}$ & $\begin{array}{l}\text { Phonoli } \\
\text { te L-Ca } \\
\text { Sodic }\end{array}$ & & $\begin{array}{l}\text { Dacit } \\
\text { e }\end{array}$ & & $\begin{array}{l}\text { Andesi } \\
\text { te }\end{array}$ & & Andesite & & $\begin{array}{l}\text { Basalti } \\
\mathrm{c} \\
\text { andesi } \\
\text { te }\end{array}$ & & $\begin{array}{l}\text { Basa } \\
\text { It }\end{array}$ & \\
\hline $\begin{array}{l}\mathrm{H}_{2} \mathrm{O} \\
\text { (range } \\
w t \%)\end{array}$ & $<0.02$ & & $\begin{array}{l}<0.0 \\
2\end{array}$ & & $<0.02$ & & $<0.02$ & & $<0.02$ & & $\begin{array}{l}<0.0 \\
2\end{array}$ & \\
\hline $\begin{array}{l}n \\
\text { Analyses }\end{array}$ & 42 & $1 \sigma$ & 3 & $1 \sigma$ & 3 & $1 \sigma$ & 3 & $1 \sigma$ & 3 & $1 \sigma$ & 3 & $1 \sigma$ \\
\hline $\mathrm{SiO}_{2}$ & 60.72 & $\begin{array}{l}0.1 \\
6\end{array}$ & $\begin{array}{l}65.1 \\
2\end{array}$ & $\begin{array}{l}0.0 \\
9\end{array}$ & 62.34 & $\begin{array}{l}0.1 \\
5\end{array}$ & 60.50 & $\begin{array}{l}0.1 \\
8\end{array}$ & 54.06 & $\begin{array}{l}0.0 \\
5\end{array}$ & $\begin{array}{l}48.5 \\
7\end{array}$ & $\begin{array}{l}0.3 \\
0\end{array}$ \\
\hline $\mathrm{TiO}_{2}$ & 0.56 & $\begin{array}{l}0.0 \\
3\end{array}$ & 0.66 & $\begin{array}{l}0.0 \\
1\end{array}$ & 0.45 & $\begin{array}{l}0.0 \\
1\end{array}$ & 0.59 & $\begin{array}{l}0.0 \\
1\end{array}$ & 0.92 & $\begin{array}{l}0.0 \\
4\end{array}$ & 1.77 & $\begin{array}{l}0.0 \\
5\end{array}$ \\
\hline $\mathrm{Al}_{2} \mathrm{O}_{3}$ & 18.89 & $\begin{array}{l}0.0 \\
9\end{array}$ & $\begin{array}{l}16.7 \\
9\end{array}$ & $\begin{array}{l}0.1 \\
3\end{array}$ & 18.36 & $\begin{array}{l}0.1 \\
0\end{array}$ & 18.15 & $\begin{array}{l}0.1 \\
7\end{array}$ & 19.23 & $\begin{array}{l}0.0 \\
3\end{array}$ & $\begin{array}{l}17.4 \\
6\end{array}$ & $\begin{array}{l}0.0 \\
9\end{array}$ \\
\hline $\mathrm{FeOa}$ & 3.32 & $\begin{array}{l}0.2 \\
0\end{array}$ & 4.33 & $\begin{array}{l}0.0 \\
9\end{array}$ & 6.29 & $\begin{array}{l}0.0 \\
6\end{array}$ & 6.34 & $\begin{array}{l}0.0 \\
4\end{array}$ & 8.21 & $\begin{array}{l}0.0 \\
8\end{array}$ & $\begin{array}{l}10.6 \\
5\end{array}$ & $\begin{array}{l}0.1 \\
5\end{array}$ \\
\hline $\mathrm{MnO}$ & 0.20 & $\begin{array}{l}0.0 \\
3\end{array}$ & 0.10 & $\begin{array}{l}0.0 \\
3\end{array}$ & 0.16 & $\begin{array}{l}0.0 \\
3\end{array}$ & 0.18 & $\begin{array}{l}0.0 \\
2\end{array}$ & 0.22 & $\begin{array}{l}0.0 \\
1\end{array}$ & 0.19 & $\begin{array}{l}0.0 \\
1\end{array}$ \\
\hline $\mathrm{MgO}$ & 0.36 & $\begin{array}{l}0.0 \\
1\end{array}$ & 2.27 & $\begin{array}{l}0.0 \\
2\end{array}$ & 2.14 & $\begin{array}{l}0.0 \\
3\end{array}$ & 2.55 & $\begin{array}{l}0.0 \\
3\end{array}$ & 3.19 & $\begin{array}{l}0.0 \\
5\end{array}$ & 5.50 & $\begin{array}{l}0.0 \\
4\end{array}$ \\
\hline $\mathrm{CaO}$ & 0.68 & $\begin{array}{l}0.0 \\
3\end{array}$ & 5.01 & $\begin{array}{l}0.0 \\
4\end{array}$ & 5.79 & $\begin{array}{l}0.0 \\
8\end{array}$ & 7.14 & $\begin{array}{l}0.0 \\
5\end{array}$ & 8.84 & $\begin{array}{l}0.0 \\
9\end{array}$ & $\begin{array}{l}10.1 \\
1\end{array}$ & $\begin{array}{l}0.2 \\
4\end{array}$ \\
\hline $\mathrm{Na}_{2} \mathrm{O}$ & 9.80 & $\begin{array}{l}0.0 \\
9\end{array}$ & 3.54 & $\begin{array}{l}0.0 \\
4\end{array}$ & 3.55 & $\begin{array}{l}0.0 \\
6\end{array}$ & 3.71 & $\begin{array}{l}0.1 \\
4\end{array}$ & 3.67 & $\begin{array}{l}0.0 \\
1\end{array}$ & 3.92 & $\begin{array}{l}0.0 \\
1\end{array}$ \\
\hline $\mathrm{K}_{2} \mathrm{O}$ & 5.47 & $\begin{array}{l}0.0 \\
6\end{array}$ & 2.19 & $\begin{array}{l}0.0 \\
4\end{array}$ & 0.93 & $\begin{array}{l}0.0 \\
2\end{array}$ & 0.84 & $\begin{array}{l}0.0 \\
2\end{array}$ & 1.66 & $\begin{array}{l}0.0 \\
7\end{array}$ & 1.83 & $\begin{array}{l}0.0 \\
1\end{array}$ \\
\hline Total & 99.58 & & $\begin{array}{l}99.4 \\
1\end{array}$ & & 100.01 & & 99.91 & & 99.08 & & $\begin{array}{l}98.0 \\
9\end{array}$ & $\begin{array}{l}0.4 \\
5\end{array}$ \\
\hline $\mathrm{NBO} / \mathrm{T}$ & 0.10 & & 0.11 & & 0.10 & & 0.15 & & 0.24 & & 0.44 & \\
\hline $\mathrm{A} / \mathrm{CNK}^{\mathrm{a}}$ & 0.81 & & 0.97 & & 1.06 & & 0.91 & & 0.80 & & 0.65 & \\
\hline
\end{tabular}




\begin{tabular}{|c|c|c|c|c|c|c|}
\hline $\begin{array}{l}\mathrm{Na}_{2} \mathrm{O}+\mathrm{K} \\
{ }_{2} \mathrm{O}\end{array}$ & 15.3 & 5.7 & 4.5 & 4.6 & 5.3 & 5.7 \\
\hline $\mathrm{K}_{2} \mathrm{O} / \mathrm{Na}_{2} \mathrm{O}$ & 0.6 & 0.6 & 0.3 & 0.2 & 0.5 & 0.5 \\
\hline${ }_{)}^{\mathrm{Al} /(\mathrm{Al}+\mathrm{Si}}$ & 0.27 & 0.23 & 0.26 & 0.26 & 0.30 & 0.30 \\
\hline \multicolumn{7}{|c|}{ Glass synthesis conditions } \\
\hline $\begin{array}{l}\text { Techniqu } \\
\mathrm{e}\end{array}$ & PC & PC & PC & PC & PC & PC \\
\hline$P$ (kbar) & 0.001 & $\begin{array}{l}0.00 \\
1\end{array}$ & 0.001 & 0.001 & 0.001 & $\begin{array}{l}0.00 \\
1\end{array}$ \\
\hline$T\left({ }^{\circ} \mathrm{C}\right)$ & 1500 & 1500 & 1500 & 1500 & 1500 & 1550 \\
\hline $\begin{array}{l}\text { Quench } \\
\text { rate } \\
\left({ }^{\circ} \mathrm{C} / \mathrm{s}\right)\end{array}$ & 200 & 200 & 200 & 200 & 200 & 200 \\
\hline
\end{tabular}

PC: piston cylinder; NBO/T: non-bridging oxygen per tetrahedron. Analytical conditions: $15.0 \mathrm{kv}$, $10 \mathrm{nA}$, defocused beam of diameter 10-20 $\mu \mathrm{m}$. References: 3: Giordano et al., 2000; 4: Giordano and Dingwell, 2003b and Giordano and Dingwell, 2003c; 5: Giordano and Dingwell, 2003a. Further data on phonolites and rhyolites are in Di Muro et al., 2006 and Arias et al., 2006. ${ }^{a}$ Molar Al203/(CaO + Na2O + K2O).

Micro-Raman measurements of natural glasses in the range $200-1800 \mathrm{~cm}^{-1}$ reveal well-defined asymmetric bands that are reproducible throughout each glass sample. In dry glasses, the most intense bands are in the range $250-650 \mathrm{~cm}^{-1}$ (low frequency band: LF) and $850-1250 \mathrm{~cm}^{-1}$ (high frequency band: HF) (Fig. 1). Significantly less intense bands are found in the domain $650-850 \mathrm{~cm}^{-1}$. The spectral distribution at $\sim$ $800 \mathrm{~cm}^{-1}$ is associated to vibration modes related to the overall response of the silicate network, and the corresponding intensity drops are associated to an increase of the depolymerization degree (Fig. 1; Sharma et al., 1997). 

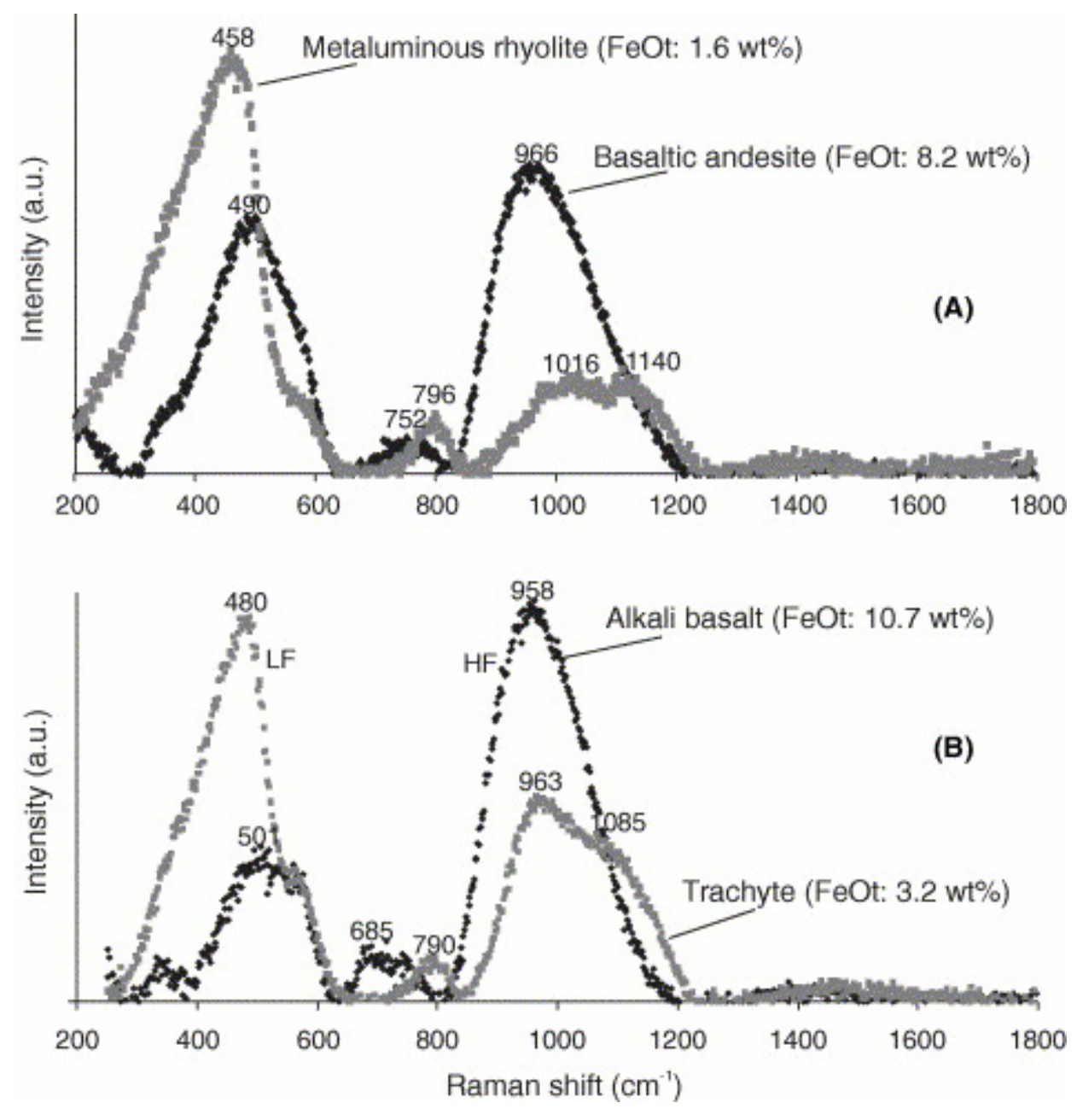

Fig. 1. Unpolarized Raman spectra (after baseline subtraction) of the remolten anhydrous glasses, whose compositions correspond to the glassy matrix of (A) metaluminous obsidian $\left(0.5 \mathrm{H}_{2} \mathrm{O}_{T}\right)$ from Lipari (Di Muro et al., 2006) and basaltic-andesite from Merapi and (B) of the dry 1538 AD Monte Nuovo trachyte pumiceous samples (Giordano et al., 2004) and the 2002 Etna trachybasalt (Giordano and Dingwell, 2003a). Note the increasing intensity of the band at $\approx 960 \mathrm{~cm}^{-1}$ as a function of iron content.

In silicate structure, the $\mathrm{LF}$ and $\mathrm{HF}$ components result from vibrations of $\mathrm{T}$ (tetrahedrally coordinated network-forming cations: $\mathrm{Si}^{4+}, \mathrm{Al}^{3+}, \mathrm{Fe}^{3+}$ ) and $\mathrm{O}$ atoms in the glass framework (McMillan, 1984, Mysen, 1988 and Sharma et al., 1997). Band intensity, frequency and spectral composition change as a function of (i) substitution in the glass framework of $\mathrm{Si}^{4+}$ by alkali-balanced $\mathrm{Al}^{3+}$ and $\mathrm{Fe}^{3+}$ in tetrahedral coordination and (ii) introduction of alkali oxides that breaks the TOT bonds and generates nonbridging oxygens (NBOs; Furukawa et al., 1981, Neuville and Mysen, 1996 and Sharma et al., 1997). In the HF region, substitution of $\mathrm{Si}^{4+}$ by $\mathrm{Al}^{3+}$ is expected to increase the scattering cross-section and to decrease the frequency of the $1000-1150 \mathrm{~cm}^{-1}$ feature. The possible existence of structural units with different inter-tetrahedral angles may also determine the spectral composition of this band (Seifert et al., 1982). Substitution of $\mathrm{Fe}^{3+}$ in tetrahedral coordination results in an 
intense band at $\sim 965 \mathrm{~cm}^{-1}$ (Sharma et al., 1997). Approximately symmetric stretching of $\mathrm{Q}^{n}$ units $\left(\mathrm{Q}\right.$ : $\mathrm{TO}_{4}$ polyhedra; $n$ : number of bridging oxygens $\mathrm{BOs}$ ) contributes to the spectral distribution of the HF region (Furukawa et al., 1981). In the LF domain $\left(460-500 \mathrm{~cm}^{-1}\right)$, the proportion of structural units (rings) formed by variable numbers of tetrahedra determines the asymmetric shape of the band and its evolution with glass composition and structure (Mysen, 1988).

In our set of natural glasses, we observe spectral evolutions consistent with the cited cationic substitutions studied in synthetic glasses of more simple composition (Fig. 1). In fact, very high intensity of the LF band $\left(460-480 \mathrm{~cm}^{-1}\right)$ is observed in highly polymerized rhyolites and trachytes and it progressively diminishes in less polymerized, iron-rich mafic glasses (Fig. 1a and b). The HF band consists of a lowintensity doublet (about $1015 \mathrm{~cm}^{-1}$ and $1140 \mathrm{~cm}^{-1}$ ) in rhyolites and it shifts to lower frequencies with increasing $\mathrm{Al} /(\mathrm{Al}+\mathrm{Si}$ ) (Fig. 1a and b). In particular, a band at 960$980 \mathrm{~cm}^{-1}$ becomes increasingly intense from sialic to mafic compositions (Fig. 1a and b).

In calcalkaline glasses, the LF/HF intensity ratio linearly decreases as a function of the $\mathrm{Al} /(\mathrm{Al}+\mathrm{Si})$ ratio and glass iron content (Fig. 2a). Al-rich alkaline glasses of Pollena and Pompei deviates from this linear trend, but also in that case the intensity of the $\sim 960 \mathrm{~cm}^{-1}$ band becomes more intense in the more iron-rich Pollena glass. The data of both alkaline and calcalkaline sets show that the TOT band ratio is also correlated with the "degree of polymerization" of the melt, expressed by the NBO/T ratio (non-bridging oxygens per tetrahedron) (Fig. $2 b$ ). The increase in intensity of the band at $\sim 960 \mathrm{~cm}^{-1}$ can originate both from asymmetric stretching of $\mathrm{Fe}-\mathrm{O}-\mathrm{Si}$ and from symmetric stretching of TO nonbridging bonds in $Q^{2}$ species (Sharma et al., 1997). However, our unpolarized spectra do not allow discriminating between these two contributions. In the range $0-10 \mathrm{wt} \%$, dissolution of water in the melt determines an additional second-order increase of the LF/HF band intensity ratio (Fig. 2b; ii Muro et al., 2006).

The band at $\sim 490 \mathrm{~cm}^{-1}$ was used for internal normalization procedures for water analysis in rhyolitic glasses (Thomas, 2000; Chabiron et al., 2004) as it is the most intense band in highly polymerized glasses. The increase in intensity of the $\sim$ $960 \mathrm{~cm}^{-1}$ band in the iron-rich and the most depolymerized melts suggests that it could be adopted to determine, using an internal calibration procedure, the water content of mafic magmas (andesites-basalts). However, these internal calibrations will be probably affected by the oxidation state of iron and the structural role assumed by iron species. 

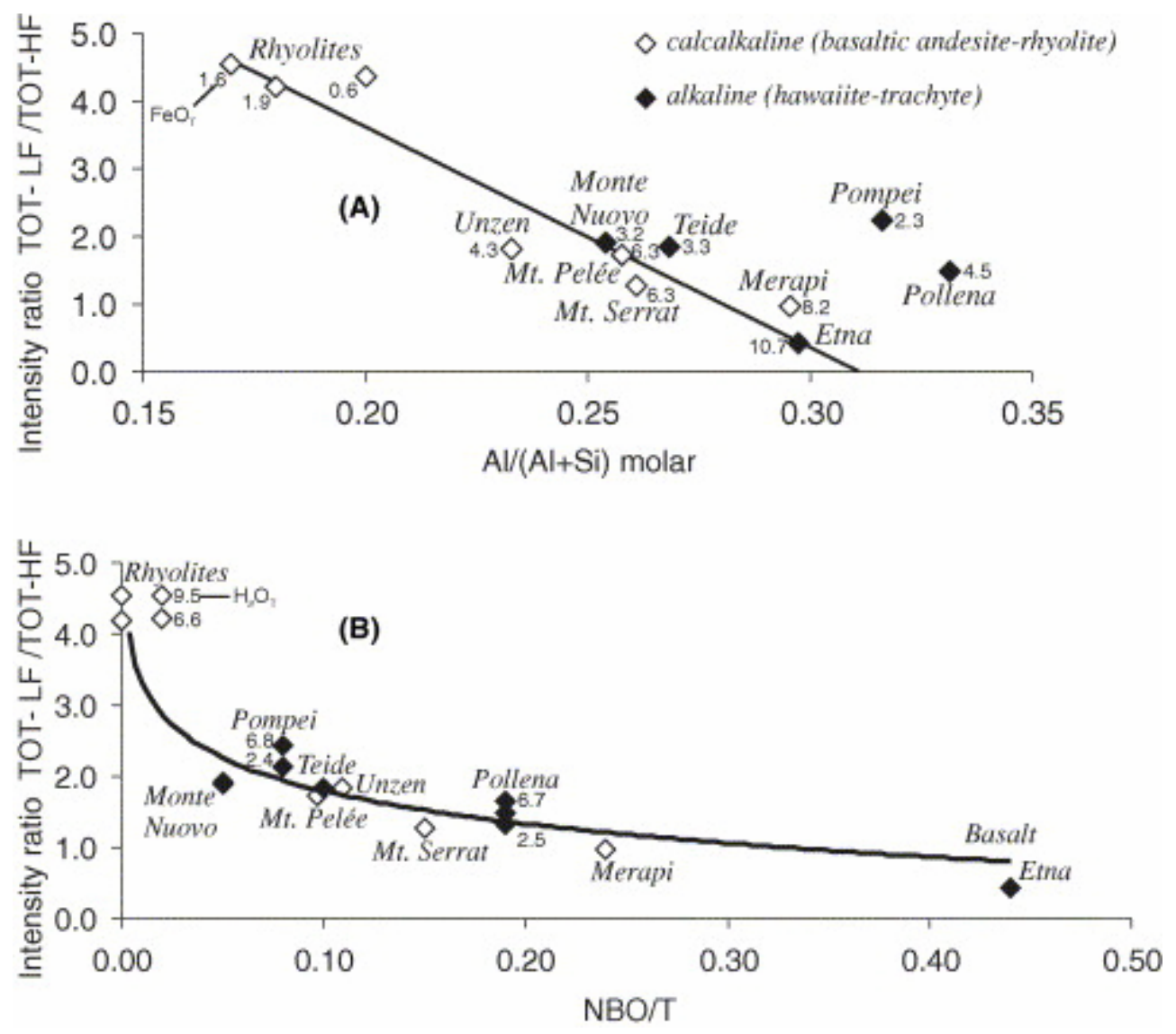

Fig. 2. Intensity ratio of the two main bands (LF: low frequency; HF: high frequency) as a function of: (A) $\mathrm{Al} /(\mathrm{Al}+\mathrm{Si})$ molar ratio and total iron content and (B) polymerization of the glasses. In order to show the influence of glass composition on band intensity ratios, NBO/T refers to the dry glass composition. Open squares: calc-alkaline magmas; filled squares: alkaline magmas. Numbers: (A) total iron content, (B) total dissolved water content. Rhyolites data are from Di Muro et al., 2006. Other compositions are in Table 1 and Table 2.

\subsection{Influence of thermal history}

Raman spectra taken in splits of trachyte samples (MNV) reveal that they have variably reacted to thermal annealing depending on their primary dissolved water contents. Appreciable changes on spectra topology are not observed in dry glasses. On the contrary, additional spectral features appear in hydrous samples that underwent some heating stage. For these samples, we observed sharp peaks at 150 , 190, 241, 330, and $670 \mathrm{~cm}^{-1}$ (Fig. 3a). The appearance of these peaks is associated to a substantial decrease in intensity of the broad bands HF and LF related to vibration of the alluminosilicate glass network (Fig. 3). It is noteworth that a strong decrease in intensity of the $\sim 960 \mathrm{~cm}^{-1}$ band, at least partly due to $\mathrm{Fe}^{3+}$ in tetrahedral coordination, is observed in concomitance with microlite crystallization. 

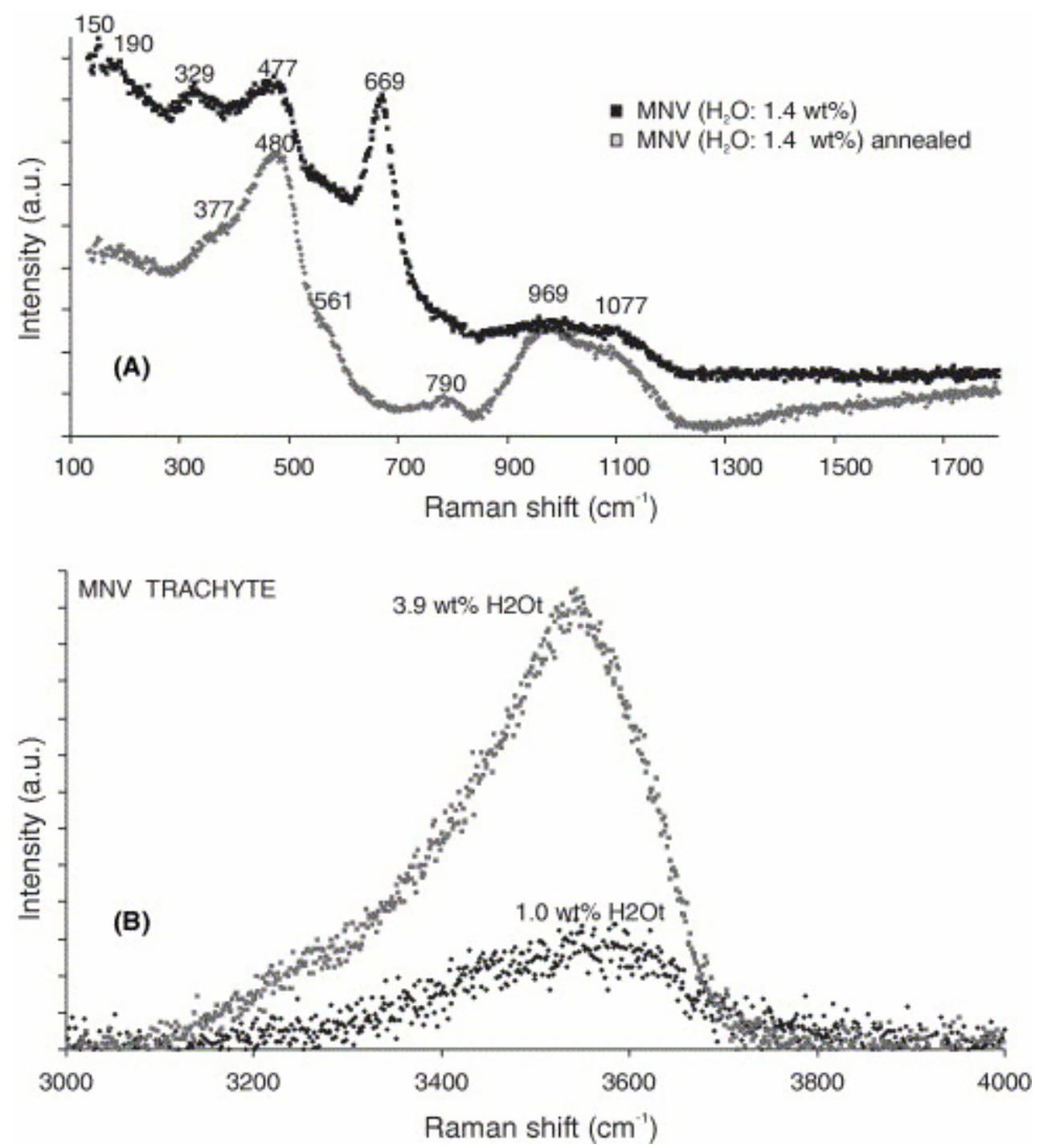

Fig. 3. Unpolarized Raman spectra of hydrous $\left(\mathrm{H}_{2} \mathrm{O}_{\mathrm{T}} 1.0 \mathrm{wt} \%\right)$ trachyte glasses from Monte Nuovo (Phlegrean Fields). (A) Appearance of sharp peaks related to iron oxide crystallization during thermal annealing and their subsequent oxidation under the laser beam is observed in the hydrous sample. A concominant decrease of the $\sim 960 \mathrm{~cm}^{-1}$ band partly related to stretching of the Fe-O-Si bonds is observed. (B) Increasing amounts of dissolved water in the glass linearly increase the intensity and the area of the $\mathrm{H}_{2} \mathrm{O}_{t}$ band and modify its spectral composition (Di Muro et al., 2006).

Decrease of the $\approx 960 \mathrm{~cm}^{-1}$ band is explained by the iron-rich nature of the microcrystals. In fact, the series of cited peaks can be correlated with presence of iron oxide (magnetite) microlites dispersed in the glassy matrix (Wang et al., 2004). In particular, the bands in the low frequency range $\left(<250 \mathrm{~cm}^{-1}\right)$ are indicative of an early oxidation stage of the magnetite microlites, possibly consequent to heating under the laser beam (Wang et al., 2004 and de Faria et al., 1997). Comparable microcrystallization of oxides has been described in annealed andesite samples (Liebske et al., 2003) and dacite glasses experiencing slow isobaric cooling (Di Muro et al., 2006). Liebske et al. (2003) proposed that the increase in viscosity at constant temperature was determined by melt structure variations produced by crystallization of iron-rich phases. Our Raman spectra suggest that the viscosity increase may be due to change of the structural role played by iron. In particular, the occurrence of oxide phases in amounts increasing as a function of dissolved water content 
indicates that bulk crystallization is controlled by an enhanced diffusion rate in the annealed hydrous glasses.

\subsubsection{Micro-crystallization and degassing: the example of obsidian glasses}

Banded obsidian glasses represent an excellent example of heterogeneity at the microscale determined by the presence of variable amounts and types of microlites and vesicles. We analyzed dissolved water content in banded (black/white) obsidians from Milos Island (Greece) (Fig. 4).

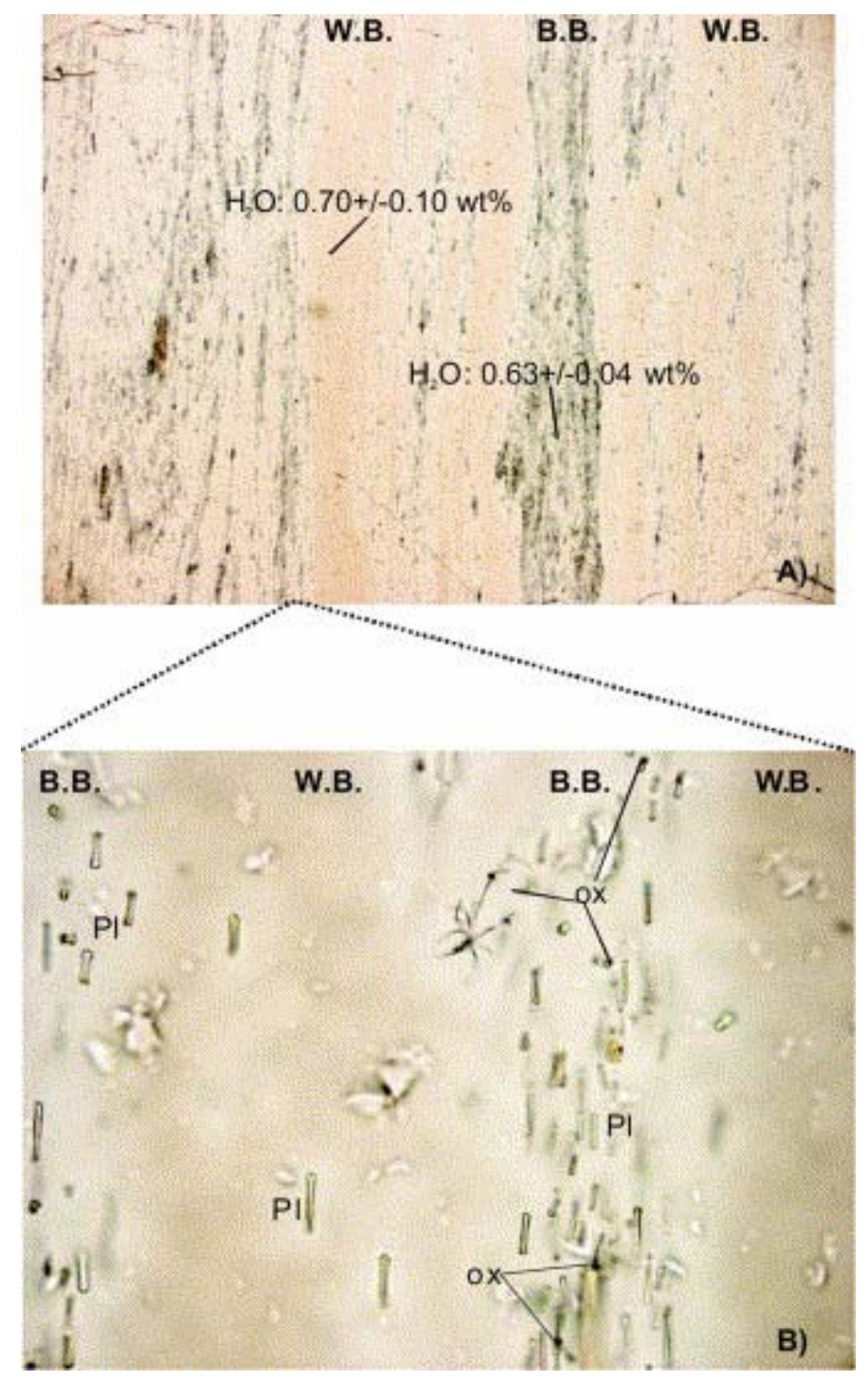

Fig. 4. (A) Microphotograph of banded obsidian from Milos (Greece) (picture height: $5 \mathrm{~mm}$ ) and (B) detail (image width: $240 \mu \mathrm{m}$ ) of white (microlite-free) and black (microlite-charged) bands. Microlites are plagioclase, amphibole and oxides and show textures typical of fast growth. Raman analyses show that no appreciable differences in dissolved water content (0.6-0.7 wt\%) are observed between the bands. That may suggest that thermal history more than degassing alone could be responsible of the observed differences in crystallinity of the glasses. Further data on obsidian composition and dissolved water content are in Arias et al. (2006). 
Dissolved water contents were determined through an external calibration procedure using a set of metaluminous obsidians and rhyolites for calibration (Di Muro et al., $\underline{2006}$ and Arias et al., 2006). The normalized intensity of the peak at $\approx 3450 \mathrm{~cm}^{-1}$ was found to linearly correlate with $\mathrm{H}_{2} \mathrm{O}_{\mathrm{T}}$. In the low frequency domain, Raman spectra show the presence of broad TOT band associated with peaks produced by the scattering of dispersed microlites. The most striking difference is observed between spectra collected in black and white layers in banded obsidians (Fig. 4a). In the black layers, a broad feature characterised by a doublet at $1360 \mathrm{~cm}^{-1}$ and $1590 \mathrm{~cm}^{-1}$ is found. This doublet can be produced by iron oxidation of magnetite under the heating of laser beam (de Faria et al., 1997). Optical observation (Fig. 4b) confirms that black layers contain a significant amount of dispersed iron oxide microlites that often act as nuclei for heterogeneous crystallization of other silicate phases (e.g. feldspars). In spite of observed differences in microlite distribution, the dissolved $\mathrm{H}_{2} \mathrm{O}_{\mathrm{T}}$ contents are not appreciably different between black (microlite-bearing) and white (microlite-free) bands (Fig. 4a). Castro and Mercer (2004) obtained similar results through FTIR analyses of banded obsidians from Inyo domes. These evidences suggest that variable magma degassing and undercooling cannot explain alone the differences in microlite distributions (Hammer et al., 1999). Following the model of Gonnermann and Manga (2003) who attribute obsidian banding to viscous shear near the walls of volcano conduit, we propose that variable magma heating and cycles of thermal annealing during shear may also influence heterogeneous crystallization in obsidians.

\section{Implications for calibration procedures}

In the previous paragraphs, we have shown that the internal normalization procedure proposed by Thomas (2000) and adopted by Chabiron et al. (2004) is markedly composition dependent and it can be affected by glass microcrystallization during thermal annealing. Di Muro et al. (2006) have shown that normalization of the total water band $\left(\mathrm{H}_{2} \mathrm{O}_{\mathrm{T}}\right)$ of a given sample to the $\mathrm{H}_{2} \mathrm{O}_{\mathrm{T}}$ of a glass standard (external procedure) strongly reduces this composition dependence. $\mathrm{H}_{2} \mathrm{O}_{\mathrm{T}}$ band is the convolution of at least four modes related to stretching of variably $\mathrm{H}$-bonded molecular water $\left(\mathrm{H}_{2} \mathrm{Om}\right)$ and hydroxyls $(\mathrm{OH})$ (Mysen et al., 1997, Chabiron et al., 2004 and Di Muro et al., 2006). The external procedure is composition-dependent as the internal one because it is influenced by the subtle changes in water speciation and $\mathrm{H}$-bonding. Glass composition (especially alkali content) and cooling history are well-known factors that determine water speciation (eg. Sylver et al., 1990 and Zhang et al., 1995). We tested the external procedure on three sets of phonolite (Pm and Po) and trachyte (MNV) hydrous glasses. For this purpose, intensity of the $\mathrm{H}_{2} \mathrm{O}_{T}$ band was measured after baseline subtraction and normalized to $\mathrm{H}_{2} \mathrm{O}_{T}$ intensity of a dacite glass standard containing $2.1 \mathrm{wt} \%$ dissolved water. A second order polynomial was adopted to fit the background (see details in Di Muro et al., 2006).

Results show that a single calibration line $\left(R^{2}: 0.988\right.$; not shown in the figure) could actually be adopted for trachytes and phonolites in spite of large variations in composition, synthesis conditions and cooling histories of this set of alkaline silicic glasses (Fig. 5). 


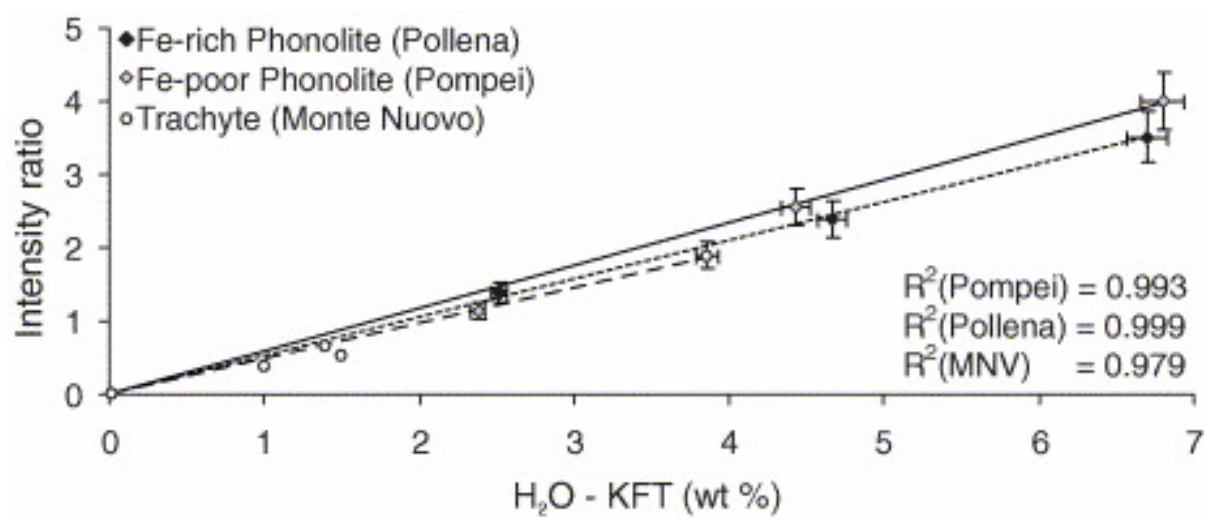

Fig. 5. Calibration line (external procedure) for determination of dissolved water content in silicic alkaline glasses. Triangles: MNV trachyte; squares: Pm phonolite; Rombs: Po phonolite.

Calibrations for Ca-rich phonolite (Pollena) and trachyte differ of 10 and $22 \%$ relative respect to the calibration defined for the Ca-poor phonolite (Pompei). These differences are smaller than the error associated to water determination using a calibrated "by difference" EPMA procedure ( $0.5 \mathrm{wt} \%$; Pichavant et al., 2002 and references therein) in phonolite glasses with $\mathrm{H}_{2} \mathrm{O}_{T}<5 \mathrm{wt} \%$ and in trachyte glasses with $\mathrm{H}_{2} \mathrm{O}_{\mathrm{T}}<2.5 \mathrm{wt} \%$. The very similar linear correlations we observe could suggest that a strong similarity exists in the evolution of water speciation in these glasses as a function of total water content.

\section{Conclusions}

Raman spectrometry allows quantitative analysis of water dissolved in glasses by the determination of the normalized height or area of the total water band in the highfrequency region $\left(3000-3800 \mathrm{~cm}^{-1}\right)$ of the spectra. Water band intensity can be normalized either to a band related to vibration of the aluminosilicate glass network (internal procedure) or to the total $\mathrm{OH}$-stretching band of a glass standard (external procedure). The internal procedures are potentially strongly dependent on melt polymerization degree and substitutions of $\mathrm{Fe}^{3+}$ and $\mathrm{Al}^{3+}$ for $\mathrm{Si}^{4+}$ in tetrahedral coordination. External procedures are mostly dependent of parameters influencing water speciation and $\mathrm{H}$-bonding of water species. However, the external procedure is only faintly composition dependent as the measurement of the height of total water band allows smoothing the effect of water speciation and bonding changes that mostly broaden this spectral feature.

Beside composition, glass thermal history results to be an important factor to be taken into account when choosing the Raman analytical procedure. We found that repeated thermal annealing of hydrous alkaline glasses (trachytes) above the glass transition promotes low-temperature crystallization of oxide microlites and affects iron distribution in the melt structure. Heat treatment enhances the atomic mobility (i.e. lower viscosity) mostly in hydrous samples having high element diffusivity thus facilitating iron oxide nucleation and growth. Heating-cooling cycles show that the crystallization process is irreversible. FTIR and EPMA data indicate that neither water (up to $3.9 \mathrm{wt} \%$ ) nor chlorine (ca. $3500 \mathrm{ppm}$ ) are exsolved in alkaline melts during 
heating and crystallization of anhydrous phases. We cannot specify at which phase of the annealing cycle oxides crystallization occurs. However, occurrence of an analogous phenomenon during slow high-pressure $(3 \mathrm{kbar})$ isobaric cooling of dacite melts at the initial temperature of $1400{ }^{\circ} \mathrm{C}$ suggests that at least part of the microcrystallization can occur during the cooling phase.

During volcanic eruptions, annealing can play an important role during welding of clastogenic lava flows (Gottsmann and Dingwell, 2002) or at conduit walls heated by magma shearing (Polacci et al., 2001, Rosi et al., 2004 and Vedeneeva et al., 2005). Absence of correlation between dissolved water content and distribution of microlites in banded obsidians from Milos (Greece) suggests that degassing is not the only process that can produce the observed heterogeneities. We propose that obsidian banding and microlites crystallization may also result from cycles of magma heating by friction during the flow of viscous magma near the conduit walls (Gonnermann and Manga, 2003).

\section{Acknowledgements}

A.D.M. has been supported by a Research Training Network fellowship funded by the European Union (Research Training Network on Volcano Dynamics). C. Principe is acknowledged for providing the glass set of Milos obsidians. We thank R. Thomas and an anonymous reviewer for their constructive reviews and P. D'Arco for stimulating observations on obsidian samples.

\section{References}

Arias et al., 2006 Arias, A., Oddone, M., Bigazzi, G., Di Muro, A., Principe, C., Norelli, P., 2006. New data for the characterisation of Milos obsidians. J. Radioanal. Nucl. Chem., in press.

Bachmann and Bergantz, 2003 O. Bachmann and G.W. Bergantz, Rejuvenation of the Fish Canyon magma body: A window into the evolution of large-volume silicic magma systems, Geology 31 (2003), pp. 789-792.

Castro and Mercer, 2004 J.M. Castro and C. Mercer, Microlite textures and volatile contents of obsidian from the Inyo volcanic chain, California, Geoph. Res. Lett. 31 (2004), p. L18605.

Chabiron et al., 2004 A. Chabiron, J. Pironon and D. Massare, Characterization of water in synthetic rhyolitic glasses and natural melt inclusions by Raman spectroscopy, Contrib. Mineral. Petrol. 146 (2004), pp. 485-492.

de Faria et al., 1997 D.L.A. de Faria, S. Venâncio Silva and M.T. de Oliveira, Raman microspectroscopy of some iron oxides and oxyhydroxides, J. Raman Spectrosc. 28 (1997), pp. 873-878.

Di Muro et al., 2006 Di Muro, A., Villemant, B., Montagnac, G., Scaillet, B., Reynard, B., 2006. Determination of water content and speciation in natural silicic glasses by confocal Raman spectrometry. Geochim. Cosmochim. Acta., in press. 
Dingwell et al., 1996 D.B. Dingwell, C. Romano and K.U. Hess, The effect of water on the viscosity of a haplogranitic melt under $\mathrm{P}-\mathrm{T}-\mathrm{X}$ conditions relevant to silicic volcanism, Contrib. Mineral. Petrol. 124 (1996), pp. 19-28.

Dingwell and Virgo, 1987 D.B. Dingwell and D. Virgo, The effect of oxidation state on the viscosity of melts in the system $\mathrm{Na}_{2} \mathrm{O}-\mathrm{FeO}-\mathrm{Fe}_{2} \mathrm{O}_{3}-\mathrm{SiO}_{2}$, Geochim. Cosmochim. Acta 51 (1987), pp. 195-205.

Furukawa et al., 1981 T. Furukawa, K.E. Fox and W.B. White, Raman spectroscopic investigation of the structure of silicate glasses. III. Raman intensities and structural units in sodium silicate glasses, J. Chem. Phys. 75 (1981), pp. 3226-3237.

Giordano et al., 2000 D. Giordano, D.B. Dingwell and C. Romano, Viscosity of a Teide phonolite in the welding interval, J. Volcanol. Geoth. Res. 103 (2000), pp. 239245.

Giordano and Dingwell, 2003a D. Giordano and D.B. Dingwell, Viscosity of hydrous Etna basalt: implications to the modeling of Plinian basaltic eruptions, Bull. Volcanol. 65 (2003), pp. 8-14.

Giordano and Dingwell, 2003b D. Giordano and D.B. Dingwell, NonArrhenian multicomponent melt viscosity: a model, Earth Planet. Sci. Lett. 208 (2003), pp. 337349.

Giordano and Dingwell, 2003c D. Giordano and D.B. Dingwell, Erratum to 'Non Arrhenian multicomponent melt viscosity: a model', Earth Planet. Sci. Lett. 221 (2004), p. 449.

Giordano et al., 2004 D. Giordano, C. Romano, P. Papale and D.B. Dingwell, The viscosity of natural silicate melts: trachytes, and comparison with basalts, phonolites, and rhyolites, Chem. Geol. 213 (2004), pp. 49-61.

Gonnermann and Manga, 2003 H.M. Gonnermann and M. Manga, Explosive volcanism may not be an inevitable consequence of magma fragmentation, Nature 426 (2003), pp. 432-435.

Gottsmann and Dingwell, 2002 J. Gottsmann and D.B. Dingwell, The thermal history of a spatter-fed lava flow: the 8-ka pantellerite flow of Mayor Island, New Zealand, Bull. Volcanol. 64 (2002), pp. 410-422.

Hammer et al., 1999 J.E. Hammer, K.V. Cashman, R.P. Hoblitt and S. Newman, Degassing and microlites crystallization during pre-climactic events of the 1991 eruption of Mt. Pinatubo, Philippines, Bull. Volcanol. 60 (1999), pp. 355-380.

Liebske et al., 2003 C. Liebske, H. Behrens, F. Holtz and R. Lange, The influence of pressure and composition on the viscosity of andesitic melts, Geochim. Cosmochim. Acta. 67 (2003), pp. 473-485. 
McMillan, 1984 P.F. McMillan, Structural studies of silicate glasses and melts: applications and limitations of Raman spectroscopy, Am. Mineral. 69 (1984), pp. 622-644.

McMillan and Remmele, 1986 P.F. McMillan and R.L. Remmele, Hydroxyl sites in $\mathrm{SiO}_{2}$ glass: A note on infrared and Raman spectra, Am. Mineral. 71 (1986), pp. $772-$ 778.

Mysen et al., 1980 B.O. Mysen, D. Virgo, W.J. Harrison and C.M. Scarfe, Solubility mechanisms of $\mathrm{H}_{2} \mathrm{O}$ in silicate melts at high pressures and temperatures: A Raman spectroscopic study, Am. Mineral. 65 (1980), pp. 900-914.

Mysen, 1988 B.O. Mysen, Structure and properties of silicate melts, Elsevier (1988).

Mysen et al., 1997 B.O. Mysen, F. Holtz, M. Pichavant, J.-M. Beny and J.-M. Montel, Solution mechanisms of phosphorous in quenched hydrous and anhydrous granitic glass as a function of peraluminosity, Geochim. Cosmochim. Acta 61 (1997), pp. 3913-3926.

Neuville and Mysen, 1996 D.R. Neuville and B.O. Mysen, Role of aluminium in the silicate network: in situ, high-temperature study of glasses and melts on the join $\mathrm{SiO}_{2}-\mathrm{NaAlO}_{2}$, Geochim. Cosmochim. Acta 60 (1996), pp. 1727-1737.

Polacci et al., 2001 M. Polacci, P. Papale and M. Rosi, Textural heterogeneities in pumices from the climactic eruption of Mount Pinatubo, 15 June 1991, and implications for magma ascent dynamics, Bull. Volcanol. 63 (2001), pp. 83-97.

Pichavant et al., 2002 Pichavant, M., Martel, C., Bourdier, J.L., Scaillet, B., 2002. Physical conditions, structure, and dynamics of a zoned magma chamber: Mount Pelée (Martinique, Lesser Antilles Arc). J. Geophys. Res. 107, B5, ECV1-27.

Romano et al., 2003 C. Romano, D. Giordano, P. Papale, V. Mincione, D.B. Dingwell and M. Rosi, The dry and hydrous viscosities of alkaline melts from Vesuvius and Phlegrean Fields, Chem. Geol 202 (2003), pp. 23-38.

Rosi et al., 2004 M. Rosi, P. Landi, M. Polacci, A. Di Muro and D. Zandomeneghi, Influence of conduit-wall shear on crystal-rich magma feeding the $800 \mathrm{yr}$ BP plinian eruption of Quilotoa volcano (Ecuador), Bull. Volcanol. 66 (2004), pp. 307-321.

Seifert et al., 1982 F.A. Seifert, B.O. Mysen and D. Virgo, Three-dimensional network structure of quench melts (glass) in the systems $\mathrm{SiO}_{2}-\mathrm{NaAlO}_{2}, \mathrm{SiO}_{2}-\mathrm{CaAl}_{2} \mathrm{O}_{4}$, and $\mathrm{SiO}_{2}-\mathrm{MgAl}_{2} \mathrm{O}_{4}, \mathrm{Am}$. Mineral. 67 (1982), pp. 696-717.

Sharma et al., 1997 S.K. Sharma, T.F. Cooney, Z. Wang and S. ad van der Laan, Raman band assignments of silicate and germanate glasses using high-pressure and high-temperature spectral data, J. Raman Spectrosc. 28 (1997), pp. 697-709.

Sylver et al., 1990 L.A. Sylver, P.D. Ihinger and E. Stolper, The influence of bulk composition on the speciation of water in silicate glasses, Contrib. Mineral. Petrol. 104 (1990), pp. 142-162. 
Thomas, $2000 \mathrm{R}$. Thomas, Determination of water contents of granite melt inclusions by confocal laser Raman microprobe spectroscopy, Am. Mineral. 85 (2000), pp. 868872.

Vedeneeva et al., 2005 E.A. Vedeneeva, O.E. Melnik, A.A. Barmin and R.S.J. Sparks, Viscous dissipation in explosive volcanic flows, Geoph. Res. Lett. 32 (2005), p. L05303.

Wang et al., 2004 A. Wang, K.E. Kuebler, L.J. Bradley and A.H. Larry, Raman spectroscopy of Fe-Ti-Cr-oxides, case study: Martian meteorite EETA79001, Am. Mineral. 89 (2004), pp. 665-680.

Zhang et al., 1995 Y. Zhang, E.M. Stolper and P.D. Ihinger, Kinetics of the reaction $\mathrm{H}_{2} \mathrm{O}+\mathrm{O}=2 \mathrm{OH}$ in rhyolitic and albitic glasses: Preliminary results, Am. Mineral. 80 (1995), pp. 593-612. 